Rainer Kühne, „Plädoyer für Atlantis", Ancient Skies 1989, Preprint Version mit Anstreichungen.

Pladoyer fur die Suche nach auderirdischen Artefakten auf der trde

\title{
Linleituns
}

Es flut voh1 nur ein Thema, um das schon wcit uber 2000 Jahren heftil geatritten wird, und zu dem angeblich dber zuok bacher vertast wurdens Aclantis. und hierbel ist die Anzahl der "Lokalisationu" fast no hoch wle die der Autoren. Uber ein ahaliches Spektrum 1 at auch die batlerung des Verainkens dieber insel vertetlt. So Ileis kudelf stedner Atlantis vor buow Jaures versinken (1), und

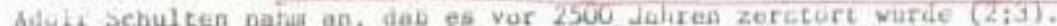

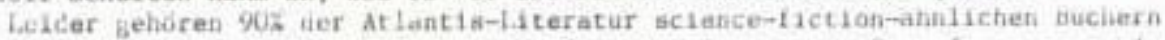
an (4). Ja, selbat die taeitien erasthaften Arbetten varatoisen oft gegen geoloyiache und archaolugische iatiachen. Andere lyyochesen viedar zeigen nur wenige Uberalnst fawimen wit dea Atlantiaberiche Platoos, daher dart es also nicite verwundera, dab dieses Theme von den orthodoxen wisenschatclern verruten wird,

\section{lier Acluncisherichic}

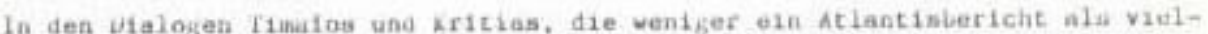

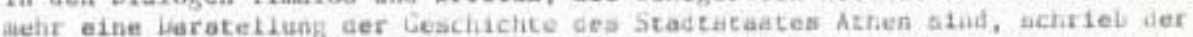

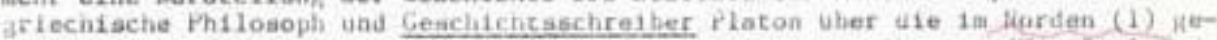
lefene Insel (Tia, 24e; T1 25 .

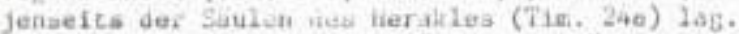

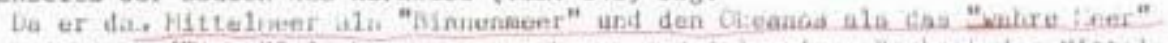

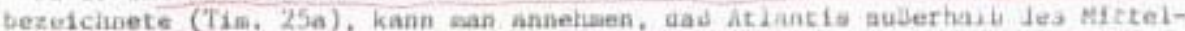

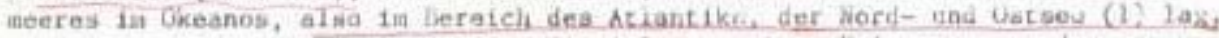

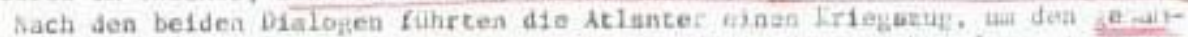

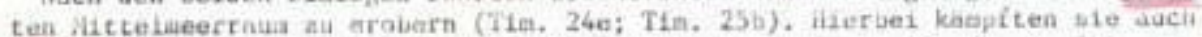

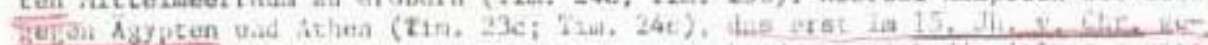

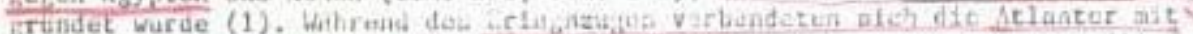

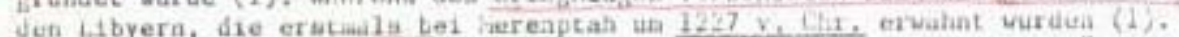

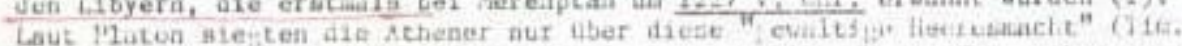

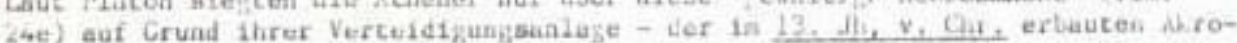

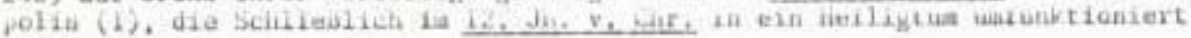
warde (1).

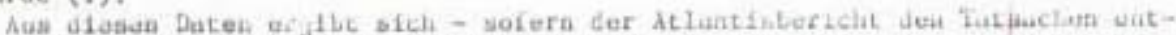

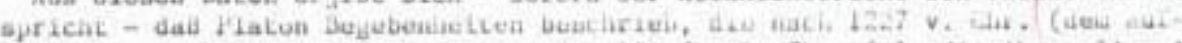
trecen der Libyar) und vor dea znde des 12. Jh. v. Chr. (ald dic Akropolis niche eohr ala Vertefdigutusanlage dfeate) atatcfandes (1).

Zahlreiche Irrcuner unterliefun, wall Platon schrieb, dail stch dieae "darchaue

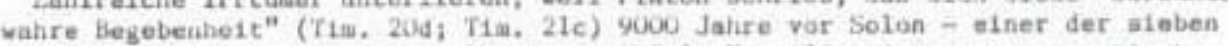

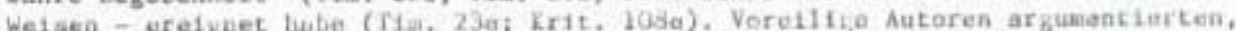
das roun Jahre vor bulon usdur haypten (ab 6 . Jt, $v$. Lif.) noch Aches uxtatiart

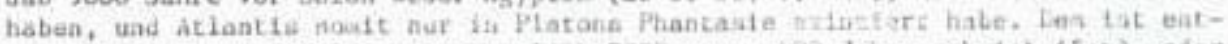
gejenzuhaiten, dals Soloa vermotientich 9000 atatt 900 Jalure schrieb $(5 ; 0)$, oder

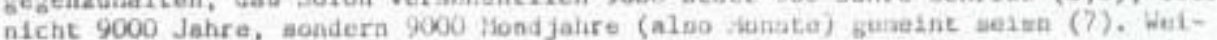

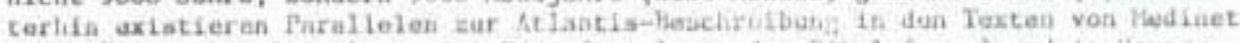
Habu/ Agypten $(1 ; 7 ; 8 ; 9)$, in der Edia $(3.4$.$) , in der Hibel (3,0$,$) und ia Howe:$ Phalakia (hierzu konate Pastor Spanuth 34 (1) Parallelen aukzeigen $(1 ; 9 ; 10)$. Rs sel noch anzunerken, daf Spanth zahl reiche srguacaio pegon die "Atlantis-hat-nicht-existiert-Hypothese" vorjelegt hat $(\theta)$.

Dhe Frage aller Fragen aber ist: Gab ea oine aas deil fiorden konsende Heerea-

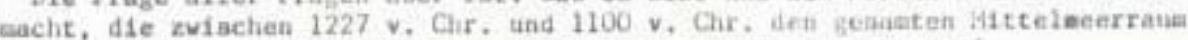
erobern wollte, abar kgypten und Athen nicht unterverfon konnte?

Diese Heereasacht lst den lifstorikern unter des kamen der lordvolker, von dumen die Schriften yon Hedinet Habu berichten $(1 ; 7 ; 9)$, gut Lekanat. Waren diese ait den Atlastern identiach?

\section{Die Nordviliker}

Tatsachlich fand Spanuth mehrere Parallelen zwtachen dea forivolkerh und dea Atlantern $(1 ; 7 ; 9)$ :

beide Staaten waren Intelin la Ukeanos, layen In forden ynd beherrachiten auch

Teile des Festlandes. Jelde Volker, deren kouIgalnsel versurihon lat, hatten den 
Plan, alle Lander des Mitteluserraumes zu erobern, wobei vor firea Kriegszus durch Vorderasien und durch Nordafrika nach Agypten ein gevaltiges Feuer (nact. Spanuth evt1. riealge Waldbranie) berzol. Sie besallen 10 Konige, elne groble Flotte, liunderttausende von Soldaten, Streitwagen (nach Spanuth ab dea 13. Jh. v. Chr, 1m Mittelaeerraui beicannt). Reiter, sowlevhafien aus Kupfer, Bisen und broize. Auberden verehrten beide Volker den Aclas.

Eeachtet man vor alles, das aie zur solbeñ Zeit auf Jiesolbe Weise Agypten angriffen, soerscheint es schwer, sie als verschiedane Volker zu betrachten.

$W 111$ man Atlantis lokalisieren, so and san beachten, dais es us $1200 \mathrm{v}$. Chr. einen Kriegszug gegen den gesanten Mittel weerraua fuhrte and eine in Norden gelegene Insel war $(3,0$,$) .$

\section{Die vichtisaten Atlantis-ilypothesen}

Wurde man sich eine Weltkarte nehaen, ua alle Urte zu kennzelchnen, an denen Atlantla verautet wurde, no gabe es wohl nur goch wentge weide Stellen. Ler leser virid daher verstehen, dab ich hier nicht alle Arlantia-Hypothesen (weit uber lou) viderlejea kana, daher konzentriere ich mich hier auf diecveventlichaten! Bach der beicanateaten Hypothese lag Atlantis fa Atlant1K $(11 ; 12 ; 13 ; 14 ; 15)$, uberreate der Insel $(12 ; 13)$ oder Inselgruppe (13) sollen die Azoren sein. Obwohl diese Hypothese so erfolgreich ist, Ubersahen alle Vertreter dieser ldee, dail Aklantis in Norden las, die Atlanter aft den Libyern verblindet waren, und Athen nur vegen soiner Acropbils siepreich war - Geachehniase alao, die Truhatena un 1227 v. Chr, stattgefunden haben konnen. Soait versank Atlantis nicht aj $5,6,04 \%$ v. Cinr. will Is Uhr Erdzeit. (12). Auch niant Huck (12) an, das der boden von Atlantis ein Kontinentalboden war, was jedoch den geologlachen rakten widerapriclit. weiterhin sind dierazoren nlcht die Uberraste eines versunikenen Kontinentes (oder oiner Insel), sondern Vulkanspitzen (1). Platon berichtete, dab das fleer biu ife versunkene Insel auch heute noch (also z, 2. Solons) vegen dus aehr seicht liejenden Schilamea unpassierbar sei (Tim. 25d). Diet trifft weniger auf die Azoran als vituehr auf das hattenweer zu (1).

Allerdiags aprechecich nicht gegen die Woglfchkeit der ehenaligen Exiatens elner "Golfstroasperre", die bis etwa dOOO V. Clir, la Atlankik bestand (12). Dieae durfte jedoch in Verlaufe der Nacheiseit kontinuierlich - keineafalls aber innerhalb von 24 Stunden (12) - versunken sein, wan vergleiche hierzu das Diagranit uber das Anstedgen des Mearesapiegels nach der Eiszeit bel biederanan (4).

Wach dem beruhaten Hellaeher KAgar Cayce lag At lantin ahe der bahana-Inael Dimini (16:17). Da die Mauern von Bindni nach den Vertretern dieser Hypothese un $10000 v$. Chr, versunken sein sollen $(4 ; 16 ; 17 ; 16)$, viderspricht dies dean Atlantioberfcht, nach dem man den Untergang der Insel fribstens ins 13. Jh. v. Chr. datteren kann $(a, 0$,$) .$

Dis bekaninteste Hypothean unseres Jahrhunderta iat die Thera-Lakalisation $(6 ; 19$;

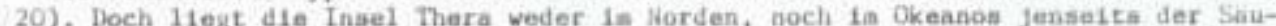
len dea Herakles, sondern in der Agafa, liach dieaer Idee beachrleb Platon dea Auar bruch des Thera ia 15. Jh. v. Chr. (6;19;20) bzw. 11ll 13. Jh. v. Chr. (1;9), bei dea die gesante Insel, also $200 \mathrm{~kat}$ Fels, gesprengt wurde. Sonit hatten - vie Spanuth benerkt (1;9) - die Xonigabur der ktInnter auf den "allseits flachen Hugel" (Krit. 113c) und thre tafenanlage (Krit. 115d) vor dea Thera-Ausbruch in 1600 w tiohe geleiten 1

Da auch Kreta weder is Norden noch in Okeanoa lag, und die Kreter des 13. JH. v. Chr. Agypten alcht angriffen, kana auch dies aicht ait atlantis identisch sein (i).

Nach Wishav, Jessen und Sichulten $(2 ; 3)$ war Atlantis ate dea phanizischen liandelahafen Tartessos bei Gibraltar Ideatiach, doch war dieser kedno label in Okeanos, Auch wurde er erat nach der Zeit Solons von den Karthagera zerstort (1).

Atlants wurde auch ifit Stonehenge gleichgesetat, doch war dieaer Megalitlibay Englanda keine Konlgsburg, von der Platon berichtet (Krit, 113c; Krit, 115et Lrit. $116 a-d ;$ Krit. $117 \mathrm{c}, \mathrm{e})$. Auberdea war in der kahe von Stonehenge ateanals ein SU Stadien vou heer entferncer liafen (kric. 115d).

Sun maj der leser jlauben, da! ich es alr bel dieser knappen "Wliferlejung" za einfach gemacht habe, daher verwelse tch auf difovicritischen Unterauchugen geitens Spanutha $(1 ; 9)$ und Cadows $(10)$. 
Spanuthe Atlantis-Theorie

We 1 ch oben geachrieben habe, waren nach Spanuth die Atlanter ait den Nordvolkera identisch. Uber dieae Volker ist nun bekannt, das die Agypter von ihnen viele Tiere erbeuteten (9). Da jedoch keine Heeresmacht Tierherden ait sich fuhrte, deutet dies darauf hin, das ea sich uil eine Vslkerwanderung handelte (9), die aus obeagenanaten Grijuden im 13. Jb. v. Chr, aus dea Norden kau. Tatwachlich ist aus disaer Zeit eine Volkerwanderuag tekanoti die Dorische, Disher wor wan der Melnung, dab dieae Nanderung threa Ursprung in Ungarn hatce, doch weist. Spanuth $(1 ; 7 ; 6 ; 9)$ darauf hin, dab san bis zug Ende des 13. Jh. v. Chr. de nordischen Griffzungenachverter und hornerhelme in Jutland, danach aber in Mittelineerraie fand. Soadt und aus veiteren genanten Critnden $(1 ; 7 ; 8 ; 9)$ folgert Spanuth, dab dieme Wanderung thren Urbprung in Jueland hatte. Aus der Gleichaetzung der Aclanter int den Nordvolkern und den Dorern folgert er weiter, dab At lantis in Jut land zu lokalisterau sei $(1 ; 7 ; 6 ; 9)$.

\section{He Lane der Lonlxainav1}

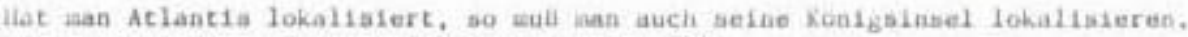
uiferzu verwendete Spausch tolgende Angabea (1):

7 bie konigainsel las la der Xundung grober flusse (krit. 1120d).

y Vor der Konigajasel lag ein hoher telaen (Lrit. 11Ga) aus roteit, weibai und schwarzent Geatein (krit. Noa), auf dem kupfererz gewoanen vurde (Krit. 114c; irit. 11ob).

* inater deiu Felsen las elae awberst fruchthare toene (Krit, 113c), In doren litte sich $9 \mathrm{ka}$ voi heer entiernt ein allselts flachar fiugel befatid (Krit. 11. euf dea die oberate burg und das hichate lieiligtua der Atlanter standen (krit. $115 \mathrm{~b})$.

- Aut dieser lasel wurde Oreichalcos (aach Spanuth gloich bernatein $(1 ; 7 ; 0 ; 9)$ ) uus dea Boden segraben, der damala (nach Spanuth ua 1200 v. Chr. $(1 ; 7 ; d ; 9)$ ) naben den cold ail hochaten deachatzt wurde (Krit. 114e).

* Nach dew Untergang der Inael wurde das Meer wegen des aehr seicht 1 iegenden Schlaimes unpasalerbar (Tia. 25d) und blieb es bis heute (Kirit. 1Gie).

Nach Spanuth $(1 ; 7 ; 6 ; 9)$ treffen diese Punkte nur auf́ das Gebtet zwtachen lielyoland und kfderatadt zu, wobel der allseits flache liugel der Steingrund lot, der heute $\theta$ anter dea Hearessplegel liegt.

Diene Theorie wurde von allen Selten beftig attackiert, doch lieì sie nur veaige Federu (b). Heute wird ain u.a. vou Gadow (10) und Kolosiao (13) anerkannt. kach Gadow (10) lat Spanuth der elnzige Atlantiaforscher, der geologische, archaologische und aycholojische Aspekte in unfangreichen Mabe berickalchtigfe.

\section{War Atlantis das Paradies?}

Ich erwhihte beraits, dal die libel Varallelen zum Atlantisbericht aufveiat. Da nach Donnelly das Paradien ait der Kontasinael der Atlanter Identisch 1st, aochte lch dieae gegenuberatellen:

1. Adaan war der erste Menach (1. Mose 2.7).

2. ix war auch ein Astronos (21).

3. ir wurde - we wuch der dak der Ldda (22) - ait aelaer trau von lott erachatien (1. Mose 2,22).

4. Wegen Uajehoraaula wurde ar aua den Paradies verbannt.

5. Nach der Vertreibung aus dem Paradiea kain ddaa in unfruchitbarea Land.

6. Adam aundigte wegen einer (atfenbar) köst11chen Erucht (1. Mose 3,6).

7. Vor der Vertreibung warea Mdau und
1. Atlas var der erate At lanter (Krit. 114a).

2. Lr war nach Plintus und biodor auch Aatronoa (1).

3. Er wurde von Poaeidon und kleite gezeugt (Xrit, 113e).

4. Lur Strafe, vell er fur die Titanen kaapfte, aubte er in die untere Kelt (23).

5. Hinter der Kondgalabel las fruchitbares Land, das ach deia teilveluea Versinken unfruchtiar wurie (1; Krit. $11 \mathrm{a}, \mathrm{b})$.

6. Wahe der conigainwel la sin Garten mit kobtilches Fruchthauaen (Krit. $115 \mathrm{~b})$.

7. Auf Atlantio herrache eif ginatiges 
tva abckt, also herrachte ein varmes Kiliaa $(24)$.

0. E1n Stroa umhab Biten (24), Da es nirgenda auf der welt eineh FIul gibt, der elne ganze Ebane ( - Eden) (24)) uagibt, handelte es sich wohl un einen Kanal.

9. In der Nitte des Paradieses befand sich der Baum der Krikenntnis $(1$, Move 2,9).

10. Eden lag in der hindung von vier grodien Flusien (1. Moue 2,10-14).
Xifina (Krit, 115; Krit, 118).

8. Eln Kanal ungab die ganze Ebene (Krit. 118c), Ubrigens wird auch von Asgard berichtet, dab es auf dea Idafeld in der Ebene Wigrid lag (9).

9. In ter Hitte Asgards (- der KundgaInael $(1 ; 11))$ befand sich die Weltenenche.

10. Die Ronigsinsel lag in der Munduag von vier sroben Flusaen (Krit, 118d).

Wle Berlitz auberdem zelgen konnte, gind Adaa und Atlas (16), aovie Atlants und Asgard (25) wortverwandt.

Ich kana hier nicht entscheiden, ob das Paradies wit Aagard und der konigainsel der Atlanter identiach 1st, doch bin ich uberzeugt, ein scichaltiges Ind12 fur Spanutha Theorie gefunden zu haben.

\section{Harallelen zu Assard}

Linen wesent11chen baustein zur Loikalisation der Kunigsinaei aieht Spanuth in deren Gleichsetzung mit Asgard, einem Keich der kdda, die bereits von Donne11y (11) Bleichgesetzt vurden. Spanuth veist auf folgende Paralleles hin (1): Asgard lag auf elner Insel, vurde voe Heer bberschweant und cauchte apacer wieder auf. Ost11ch von Aagard befand sich Peatland und Aagard aelbat las ia Wattenaeer dec. Noriaes. Auch Lst Spanuth uberzeugt, dad beatiante Textatellen den bernstein nennen, der nach iha ait don Oreichalcos der Atlanter Identisch ist. (s,o.). Die Keschrelbungen des Finbulviaters, der krdbeben und den lierichit, das dia fitze (Surt bzu. Sutur) aus dea Suden kaa, deutet er als Beschreibungen der Vorgange un $1200 \mathrm{v}$. Chr,

Dhae grundsatzitch in allen Punkten ait Spanuth wereinzustimuen, muis feh trotzdea festatellen, dab es nur schwer erklarbar ist, wenn zvel heilige "Reiche" (Asgard und Lden) in unaittelbarer Wahe lagen, ohne Ifentioch zubueill. Ich glaube aus alleif Fritier genanten Grüden folvern zu konnen, dab die konigarFisel der Atlanter mit Asgard und deal Faradies fdentiach gewesen sein konnte,

\section{hie kana man dies boweisen?}

Ich hoffe, dā̄ tch ia Verlaufe dieaes Artikela Uberzeugend gezeligt habe, dafi en wahrscheinlich ist, dab die Konigsineel der f́tanter, Kagard und Eden auf dea Steingrund dotifch von liel foland gelegen haben. Doch wfe lat dien zu beweisen? Nun, sofern - vie die Edda lerichtet (22) - Aasard nach der Zerstorung ziealich schne11 voe Sand bodeckt wurde, halte 1 ch ea a1cht fur unabjlich, d1e jeata der

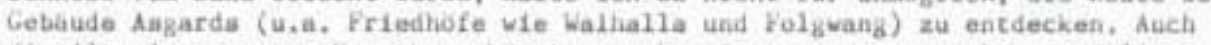

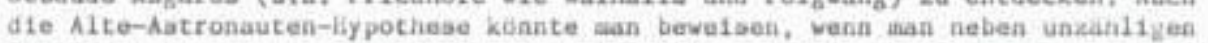
Skeletten von Menschen auch dte der Auberirdiachen entdecken wirde - ian denke 2.b. an Balders Melfahrt (22). Zu den Chancen einer bolchen kntiecicuas sei orwahnt, dai bereits die kosteneparead ausgerusteten Tauchexpeditionen seiteas Spanuths erate Lrfol be erzielten - so vurden Kauerresce, Filesenplatten and eft Elefantenutodxaho entdeckt $(1 ; 4)$. Eine bessor auagerubtate fxpedition wirie alcher noch grodere Erfolge erxielen (4) I 
Guellenverzeichinss

(1) Spanuth, Jurgen: D1e Atlahter- Volk aus dea Jernateinland; Tubingan 1974

(2) Schulten, Adolfi Atlantis; Berlin 1930

(3) Schultan, Adolf: Tertessoo, Beitrag zur ulteaten Geschichte des Westens: Hamburg 1950

(4) Bloderiaann, lians: Die versunkenen Lander; 1970

(5) Velikovsky, Lmanueli Weltea La Zusaaaenatod; Stuctgart 1950

(b) Galanopoulua, Angelost Die Wahrheit uber Atlantiaf 1960

(7) Spanuth, Jurgen: Das entritaelte Atlantis; Stuttpart 1953

(a) Spanuth, Jurgea: Und dochs Aclantis entratselt; Stuttigart 1955

(9) Spanuth, Jurgent Atlantia - Heinat, Reich und Schicksal der Gerasnea; Tutbingen 1965

(10) Gadow, Gerhard; Ler Aclantia-Streit - Zur meistdiakutierten Sage des Altertums; 1973

(11) Donnelly, Igatius: Atlantis, die vorsintflutliche kelt; tdingen 19111

(12) Huck, Otto: Allos uber Atlantia - Alte Thesen - neue Forachungen: Dusseldort 1974

(13) Koloaimo. Peter: Ste kamen von efnem anderen Stern: 1909

(14) Charroux, Kobert: Vergessene Welteat Disaeldorf 1974

(15) Blumrich, Joseph: Kasskara und die Gleben weltan - Die Geschichte der Henachheit in der itber11eferus der Hoph-1ndianer; Dusseldorf 1979

(10) Lerlitz, Charles: Geheianisse versunkener Welten; Frankfurt 1973

(17) Berlitz, Charles: Das Atlant1s-Katsel; Hunchen 1970

(13) Daniken, Krich von: Krinneruagen an die Zukunft - Ungeloste Ratsel der Vergangenheit: Dusseldorf 1968

(19) Luce, J. V.: Atlantis - Legende und Wirk11chkoit; Berk1sch-Gladbach 197s

(20) Aavor, Jaiges: Relse nach Atlant1s - Wiasenachaftler lösen das Ratsel einer Weltkatastrophe; then 1973

(21) Laniken, krich von: Strategle der Gotter - Das achte keltwunder; Dusseldorf 1982

(22) Peterich, Lckart: Gutter und Helden der Geraanen - Kleine Hythologle: 1955

(23) Kohlenberg, Kar1; Entrataelte Vorze1t; 1970

(24) Asimov, Isaac: Genea1a:- Schopfungabericht und Urzeit la Wideratrelt von Wissenachaft und Offenberung: 1903

(25) Berlitz, Charles: Spurlos - Neues aus dew Berauda-Dreieck; Muachen 1977 Samtliche Bibelzitate entaatian $1 \mathrm{ch}$ aus: Die Gute Nachrichit - Altea und Neues Testabent; Stutchart 1970 